Вестник КРАУНЦ. Физ.-мат. науки. 2021. Т. 34. №1. С. 19-28. ISSN 2079-6641

УДК $517.95+517.956 .6$

Научная статья

\title{
Об одной краевой задаче для смешанного уравнения с тремя плоскостями изменения типа в бесконечной призматической области
}

\section{Б. И. Исломов ${ }^{1}$, Г. Б. Умарова ${ }^{2}$}

1 Национальный Университет Узбекистана им. М. Улугбека, г. Ташкент, Вузгородок, ул. Университетская, д. 4, 100174, Республика Узбекистан

2 Кокандский государственый педагогический Институт им. Мукими, г. Коканд, ул. Турон, д. 23, 150700, Республика Узбекистан.

E-mail: islomovbozor@yandex.com, guzalxon5111987@gmail.com

В данной работе в бесконечной призматической области формулируется и изучается одна задача для параболо-гиперболического уравнения с тремя плоскостями изменения типа. Основным методам исследования поставленной задачи является преобразование Фурье. Доказана единственность и существование решения поставленной задачи

Ключевые слова: уравнение с тремя плоскостями изменения типа, преобразование Фурье, регулярное решение, принции экстремума, оценка решения

DOI: $10.26117 / 2079-6641-2021-34-1-19-28$

Поступила в редакцию: 26.01.2021

В окончательном варианте: 06.03.2021

Для цитирования. Исломов Б. И., Умарова Г. Б. Об одной краевой задаче для смешанного уравнения с тремя плоскостями изменения типа в бесконечной призматической области // Вестник КРАУНЦ. Физ.-мат. науки. 2021. Т. 34. № 1. С. 19-28. DOI: 10.26117/2079-66412021-34-1-19-28

Контент публикуется на условиях лищензии Creative Commons Attribution 4.0 International (https://creativecommons.org/licenses/by/4.0/deed.ru)

(С) Исломов Б. И., Умарова Г.Б., 2021

\section{Введение}

Трехмерные аналоги задаче Трикоми и Геллерстедта для уравнения эллиптикогиперболического и параболо-гиперболического типов с одной плоскостями изменения типа изучены в работах [1]-[8].

В работах $[9,10]$ изучены трехмерные задачи для параболо-гиперболического уравнения с двумя плоскостями изменения типа.

Заметим, что трехмерные задачи для параболо-гиперболического уравнения с тремя плоскостями изменения типа не изучены.

В настоящей работе изучается аналог задачи Трикоми для парабологиперболического уравнения с тремя плоскостями изменения типа в бесконечной призматической области.

Финансирование. Исследование выполнялось без финансовой поддержки фондов. 


\section{Постановка задачи}

Рассмотрим уравнение

$$
\frac{1-\operatorname{sign}(x y(1-x))}{2} U_{y y}+\frac{1+\operatorname{sign}(x y(1-x))}{2} U_{y}-U_{x x}-U_{z z}=0
$$

в трехмерной области $\Omega$, ограниченной поверхностями: $S_{0}: y=1,0 \leq x \leq 1, z \in \mathbb{R}$, $S_{1}: x+y=0, \quad y \leq 0, \quad 0 \leq x \leq \frac{1}{2}, \quad z \in \mathbb{R}, S_{2}: x-y=1, \quad y \leq 0, \quad \frac{1}{2} \leq x \leq 1, \quad z \in \mathbb{R}$, $S_{3}: y+x=0, \quad x \leq 0, \quad 0 \leq y \leq \frac{1}{2}, \quad z \in \mathbb{R}, S_{4}: y-x=1, \quad x \leq 0, \quad \frac{1}{2} \leq y \leq 1, \quad z \in \mathbb{R}$, $S_{5}: x-y=1, \quad x \geq 1, \quad 0 \leq y \leq \frac{1}{2}, \quad z \in \mathbb{R}, \quad S_{6}: x+y=2, \quad x \geq 1, \quad \frac{1}{2} \leq y \leq 1, \quad z \in \mathbb{R}$.

Введем обозначения:

$$
\begin{gathered}
J_{1}=\{(x, y, z): 0<x<1, \quad y=0, \quad z \in \mathbb{R}\}, J_{2}=\{(x, y, z): x=0,0<y<1, \quad z \in \mathbb{R}\}, \\
J_{3}=\{(x, y, z): x=1, \quad 0<y<1, \quad z \in \mathbb{R}\}, \Omega_{0}=\Omega \cap\{x>0, \quad y>0, \quad z \in \mathbb{R}\}, \\
\Omega_{1}=\Omega \cap\{x>0, \quad y<0, \quad z \in \mathbb{R}\}, \Omega_{2}=\Omega \cap\{x<0, \quad y>0, \quad z \in \mathbb{R}\}, \\
\Omega_{3}=\Omega \cap\{x>1, \quad y>0, \quad z \in \mathbb{R}\}, \Omega=\Omega_{0} \cup \sum_{i=1}^{3}\left(\Omega_{i} \cup J_{i}\right), \\
D=\Omega \cap\{z=0\}, D_{i}=\Omega_{i} \cap\{z=0\}, i=\overline{0,3}, \\
I_{m}=J_{m} \cap \quad\{z=0\},(m=\overline{1,3}) \sigma_{j}=S_{j} \cap\{z=0\}, \quad(j=\overline{0,6}), \\
A_{1}(0,0)=\bar{I}_{1} \cap \bar{I}_{2}, \quad A_{2}(1,0)=\bar{I}_{1} \cap \bar{I}_{3}, A_{3}(1,1)=\bar{I}_{3} \cap \bar{\sigma}_{0}, \quad A_{4}(0,1)=\bar{I}_{2} \cap \bar{\sigma}_{0} .
\end{gathered}
$$

Задача $A_{1} T$. Найти функцию $U(x, y, z)$ со следующими свойствами:

1) $U(x, y, z)$ непрерывна вплоть до границы области $\Omega$;

2) $U(x, y, z) \in C^{1}\left(\Omega \cup S_{2} \cup \cup S_{3} \cup S_{6}\right) \cap C_{x, y, z}^{2,1,2}\left(\Omega_{0}\right) \cap C_{x, y, z}^{2,2,2}\left(\Omega_{1} \cup \Omega_{2} \cup \Omega_{3}\right)$ и удовлетворяет уравнению (1) в областях $\Omega_{m}(m=\overline{0,3})$;

3) $U(x, y, z)$ удовлетворяет условиям

$$
\begin{gathered}
\left.U\right|_{S_{2}}=\Psi_{1}(x, z), \quad \frac{1}{2} \leq x \leq 1,\left.\quad U\right|_{S_{3}}=\Psi_{2}(y, z), \quad 0 \leq y \leq \frac{1}{2}, \quad z \in \mathbb{R}, \\
\left.U\right|_{S_{6}}=\Psi_{3}(y, z), \quad \frac{1}{2} \leq y \leq 1, \quad z \in \mathbb{R}, \\
\lim _{|z| \rightarrow \infty} U(x, y, z)=\lim _{|z| \rightarrow \infty} U_{x}(x, y, z)=\lim _{|z| \rightarrow \infty} U_{y}(x, y, z)=\lim _{|z| \rightarrow \infty} U_{z}(x, y, z)=0,
\end{gathered}
$$

где $\Psi_{1}(x, z), \Psi_{2}(y, z), \quad \Psi_{3}(y, z)$ - заданные достаточно гладкие функции, причем

$$
\lim _{|z| \rightarrow \infty} \Psi_{1}(x, z)=0, \quad \lim _{|z| \rightarrow \infty} \Psi_{2}(y, z)=\lim _{|z| \rightarrow \infty} \Psi_{3}(y, z)=0 .
$$

Решение задачи $A_{1} T$ будем искать в классе функций, представимых интегралом Фурье

$$
U(x, y, z)=\frac{1}{\sqrt{2 \pi}} \int_{-\infty}^{+\infty} u(x, y ; \lambda) e^{-i \lambda z} d \lambda
$$


тогда уравнение (1) сводится к следующему уравнению

$$
\frac{1-\operatorname{sign}(x y(1-x))}{2} u_{y y}+\frac{1+\operatorname{sign}(x y(1-x))}{2} u_{y}-u_{x x}+\lambda^{2} u=0
$$

В силу (6) задача $A_{1} T$ эквивалентно редуцируется к следующей плоской задаче.

Задача $A_{1} T_{\lambda}$. Определить в области $D$ функцию $u(x, y, \lambda)$, обладающую свойствами:

1) $u(x, y, \lambda) \in C(\bar{D}) \cap C^{1}\left(D \cup \sigma_{2} \cup \sigma_{3} \cup \sigma_{6}\right)$;

2) $u(x, y, \lambda) \in C_{x, y}^{2,1}\left(D_{0}\right) \cap C_{x, y}^{2,2}\left(D_{1} \cup D_{2} \cup D_{3}\right)$ и удовлетворяет уравнению (7) в областях $D_{m} \quad(m=\overline{0,3})$;

3) $u(x, y, \lambda)$ удовлетворяет условиям

$$
\begin{gathered}
\left.u\right|_{\sigma_{2}}=\psi_{1}(x, \lambda), \quad \frac{1}{2} \leq x \leq 1 ;\left.u\right|_{\sigma_{3}}=\psi_{2}(y, \lambda), \quad 0 \leq y \leq \frac{1}{2} ; \quad \lambda \in \mathbb{R}, \\
\left.u\right|_{\sigma_{6}}=\psi_{3}(y, \lambda), \quad \frac{1}{2} \leq y \leq 1 ; \quad \lambda \in \mathbb{R},
\end{gathered}
$$

где $\psi_{1}(x, \lambda), \quad \psi_{2}(y, \lambda), \quad \psi_{3}(y, \lambda)-$ заданные функции

$$
\psi_{n}(t, \lambda)=\frac{1}{\sqrt{2 \pi}} \int_{-\infty}^{+\infty} \Psi_{n}(t, z) e^{i \lambda z} d z, \quad(n=\overline{1,3})
$$

причем

$$
\begin{gathered}
\psi_{1}(x, \lambda) \in C\left[\frac{1}{2} ; 1\right] \cap C^{2}\left(\frac{1}{2} ; 1\right) \\
\psi_{2}(y, \lambda) \in C\left[0 ; \frac{1}{2}\right] \cap C^{2}\left(0 ; \frac{1}{2}\right), \quad \psi_{3}(y, \lambda) \in C\left[\frac{1}{2} ; 1\right] \cap C^{2}\left(\frac{1}{2} ; 1\right)
\end{gathered}
$$

и эти функции при больших значениях $|\lambda|$ удовлетворяют оценкам:

$$
\begin{array}{r}
\psi_{2}(y, \lambda)=O\left(1 /|\lambda|^{k+1} \operatorname{ch}|\lambda| \operatorname{th} \frac{|\lambda|}{2}\right), \quad \psi_{2}^{\prime}(y, \lambda)=O\left(1 /|\lambda|^{k} \operatorname{ch}|\lambda|\right), \\
\psi_{j}(t, \lambda)=O\left(\operatorname{ch} \frac{|\lambda|}{2} /|\lambda|^{k+1} \operatorname{ch}|\lambda| \operatorname{th} \frac{|\lambda|}{2}\right), \quad \psi_{j}^{\prime}(t, \lambda)=O\left(\operatorname{ch} \frac{|\lambda|}{2} /|\lambda|^{k} \operatorname{ch}|\lambda|\right),
\end{array}
$$

где $t=x$ при $j=1, t=y$ при $j=3$.

\section{Единственность и существование решения задачи $A_{1} T$}

Чтобы доказать единственность решения задачи $A_{1} T$ для уравнения (1), сначала докажем единственность решения задачи $A_{1} T_{\lambda}$ для уравнения (7).

Заметим, что для коэффициентов уравнения (7) в областях $D_{j}(j=\overline{1,3})$ известные условия Агмона-Ниренберга-Проттера [11] не выполняется. Поэтому доказать единственность решения задачи $A_{1} T_{\lambda}$ с помощью принципа экстремума сразу не удается. Несмотря на это, при помощи некоторого приема [5] можно доказать единственность решения задачи $A_{1} T_{\lambda}$. 
Введем новую неизвестную функцию $w(x, y, \lambda)$ по формуле

$$
u(x, y, \lambda)=w(x, y, \lambda) \exp \left\{\int g_{k}(\xi, \lambda) d \mu\right\}
$$

где $g_{k}(\xi, \lambda)$ - непрерывно дифференцируемое и неотрицательное решение на $[0,1]$ дифференциального уравнения

$$
g_{k}^{\prime}(\xi, \lambda)+g_{k}^{2}(\xi, \lambda)-\lambda^{2}=0, \quad(k=\overline{0,3}),
$$

здесь $\xi=x \quad$ при $\quad x>0, \quad y>0, \quad k=0 ; \quad \xi=x \quad$ при $x>0, \quad y<0, \quad k=1$; $\xi=y \quad$ при $x<0, \quad y>0, \quad k=2 ; \quad \xi=y \quad$ при $x>1, \quad y>0, \quad k=3$.

Функция $w(x, y, \lambda)$ является решением уравнения

$$
\begin{aligned}
& L_{k} w \equiv-w_{x x}+\frac{1-\operatorname{sign}(x y(1-x))}{2}\left[w_{y y}-2 g_{k}(\xi, \lambda) w_{\xi}\right]+ \\
& +\frac{1+\operatorname{sign}(x y(1-x))}{2}\left[w_{y}-2 g_{k}(\xi, \lambda) w_{\xi}\right]=0, \quad(k=\overline{0,3}) .
\end{aligned}
$$

Отметим, что для коэффициентов уравнения (15) в областях $D_{k}(k=\overline{1,3})$ известные условия Агмона-Ниренберга-Проттера выполняются. Функций $g_{k}(\xi, \lambda)$ с требуемыми свойствами может служить функция

$$
g_{k}(\xi, \lambda)=|\lambda| t h|\lambda| \xi
$$

Тогда при доказательстве единственности решения задачи $A_{1} T_{\lambda}$ важную роль играет следующий принцип максимума.

Теорема 1. Пусть $w(x, y, \lambda)$ функция обладаютщая свойствами:

1) $w(x, y, \lambda) \in C(\bar{D}) \cap C^{1}\left(D \cup \sigma_{2} \cup \sigma_{3} \cup \sigma_{6}\right)$;

2) $w(x, y, \lambda)$ функция удовлетворяет в $D_{1}$ неравенству $L_{1}[w] \leq 0$, а в $D_{j}$ неравенству $L_{j}[w] \geq 0(j=\overline{1,3})$;

3) $w(x, y, \lambda)$ является монотонно неубывающей функцией от $y, x$ и $x$ на характеристиках $\sigma_{2}, \sigma_{3}$ и $\sigma_{6}$ соответственно, т.е.

$$
\begin{gathered}
w_{x}(1+y, y, \lambda)+w_{y}(1+y, y, \lambda) \geq 0 \quad \text { на } \quad \sigma_{2} \\
w_{x}(x,-x, \lambda)-w_{y}(x,-x, \lambda) \geq 0 \quad \text { на } \quad \sigma_{3}
\end{gathered}
$$

$u$

$$
w_{x}(x, 2-x, \lambda)-w_{y}(x, 2-x, \lambda) \geq 0 \quad \text { на } \quad \sigma_{6}
$$

тогда положительный максимум функции $w(x, y, \lambda)$ в области $\bar{D}$ достигается в mочках $A_{j} \quad(j=\overline{1,3})$.

Доказательство. В силу принципа максимума для параболических уравнений [6], [12] решение $w(x, y, \lambda)$ уравнения (15) достигает своего положительного максимума в замкнутой области $\bar{D}_{0}$ на отрезках $\bar{I}_{j}(j=\overline{1,3})$. Покажем, что решение $w(x, y, \lambda)$ уравнения (15) не достигает своего положительного максимума на интервалах $I_{j}(j=\overline{1,3})$ и в точке $A_{4}(0,1)$. Предположим обратное. Пусть $w(x, y, \lambda)$ в некоторой точке $P\left(x_{0}, 0\right) \in I_{1}$ достигает свой положительный максимум. В силу непрерывности $w(x, y, \lambda), w_{y}, w_{x x}$ с учетом неравенства $L_{1}[w] \leq 0$ в (7) переходя к пределу при $y \rightarrow+0$ и получим

$$
v_{1}(x, \lambda)-\tau^{\prime \prime}{ }_{1}(x, \lambda)+\lambda^{2} \tau_{1}(x, \lambda) \leq 0, \quad(x, 0) \in I_{1},
$$


где

$$
\begin{gathered}
\tau_{1}(x, \lambda)=u(x,+0, \lambda)=w(x,+0, \lambda) \exp \left\{\int g(x, \lambda) d x\right\}, \\
v_{1}(x, \lambda)=u_{y}(x,+0, \lambda)=w_{y}(x,+0, \lambda) \exp \left\{\int g(x, \lambda) d x\right\} .
\end{gathered}
$$

Из (19) в точке $P\left(x_{0}, 0\right) \in I_{1}$ положительного максимума с учетом $\tau_{1}\left(x_{0}, \lambda\right)>0$, $\tau^{\prime \prime}{ }_{1}\left(x_{0}, \lambda\right) \leq 0$ имеем

$$
v_{1}\left(x_{0}, \lambda\right)<0, \quad\left(x_{0}, 0\right) \in I_{1} .
$$

На основании известной теоремы [11] и используя условия 1) и 2) теоремы 1 с учетом (16) в точке $P\left(x_{0}, 0\right) \in I_{1}$ положительного максимума имеем $v_{1}\left(x_{0}, \lambda\right)>0$ при $x_{0} \in(0,1)$. Это неравенство противоречит неравенству $(20)$.

Таким образом, $w(x, y, \lambda)$ не достигает своего положительного максимума на интервале $I_{1}$.

Точно также как выше используя условия 2) и 3) теоремы 1 с учетом (17), (18) можно доказать, что $w(x, y, \lambda)$ не достигает своего положительного максимума на интервалах $I_{i}(i=2,3)$. При помощи метода работы [13] можно установить, что $w(x, y, \lambda)$ не достигает своего положительного максимума в точке $A_{4}(0,1)$.

Следовательно, функция $w(x, y, \lambda)$ свой положительный максимум в области $\bar{D}$ принимает в точках $A_{j} \quad(j=\overline{1,3})$.

Теорема 1 доказана.

Теорема 2. Пусть $\psi_{2}(y, \lambda) \equiv 0, \forall y \in\left[0, \frac{1}{2}\right], \psi_{j}(t, \lambda) \equiv 0, \forall t \in\left[\frac{1}{2}, 1\right],(j=1,3), \lambda \in \mathbb{R}$, тогда если в области $D$ решение задачи $A_{1} T_{\lambda}$ для уравнения (7) существует, то оно единственно.

Доказательство. Пусть $\psi_{1}(x, \lambda) \equiv 0, \psi_{2}(y, \lambda) \equiv 0, \quad \psi_{3}(y, \lambda) \equiv 0, \quad$ тогда на основании теоремы 1 с учетом (14) имеем $w(x, y, \lambda) \equiv 0$ в $\bar{D}_{0}$. В силу единственности решения задачи Коши [9] в области $D_{j}(j=\overline{1,3})$ для уравнения (15) получаем, что $w(x, y, \lambda) \equiv 0$ в $\bar{D}_{j}(j=\overline{1,3})$. Отсюда следует, что

$$
w(x, y, \lambda) \equiv 0 \quad \text { в } \quad \bar{D} .
$$

Следовательно, в силу (21) из (14) имеем

$$
u(x, y, \lambda) \equiv 0 \quad \text { в } \quad \bar{D} .
$$

Тем самым, решение задачи $A_{1} T_{\lambda}$ единственно. Теорема 2 доказана.

Для обеспечения существования интеграла (6) и выполнения условий находим, оценку решения задачи $A_{1} T_{\lambda}$ при больших значениях параметра $|\lambda|$.

\section{Имеет место.}

Теорема 3. Если выполнены условия (13), то функция и $(x, y, \lambda)$ при больших значениях $|\lambda|$ допускает оценку

$$
u(x, y, \lambda)=O\left(1 /|\lambda|^{k}\right), \quad k>3 .
$$

Доказательство. Теорема 3 доказывается с помощью принципа экстремума [6, C. 10] для параболических и гиперболических уравнений и при помощи метода работы [5]. Единственность решения задачи $A_{1} T$ следует из теоремы 2 с учетом (6) и (10). 
Существование решения задачи $A_{1} T$ доказывается методом интегральных уравнений. Чтобы доказать существование решения задачи $A_{1} T$, сначала доказывается существование решения задач $A_{1} T_{\lambda}$ для уравнения (7).

Теорема 4. Если выполнены условия (11) и (12), то в области D решение задачи $A_{1} T_{\lambda}$ для уравнения (7) существует.

Доказательство.

В силу условий 1$)$ - 2) задачи $A_{1} T_{\lambda}$, переходя к пределу при $y \rightarrow+0$ в уравнении $(7)$, с учетом $(8),(9)$ как в работе $[6$, C. 110$]$ из области $D_{0}$ на $I_{1}$ получим

$$
\begin{gathered}
\tau^{\prime \prime}{ }_{1}(x, \lambda)-\lambda^{2} \tau_{1}(x, \lambda)=v_{1}(x, \lambda), \quad(x, 0) \in I_{1}, \\
\tau_{0}(0, \lambda)=\psi_{2}(0, \lambda), \quad \tau_{0}(1, \lambda)=\psi_{1}(1, \lambda),
\end{gathered}
$$

где

$$
\tau_{0}(x, \lambda)=u(x,+0 ; \lambda), \quad(x, 0) \in \bar{I}_{1}, \quad v_{0}(x, \lambda)=u_{y}(x,+0, \lambda), \quad(x, 0) \in I_{1} .
$$

Решая задачу (24) и (25) получим функциональное соотношение между $\tau_{0}(x, \lambda)$ и $v_{0}(x, \lambda)$, перенесенное из области $D_{0}$ на $I_{1}$ :

$$
\tau_{0}(x, \lambda)=\int_{0}^{1} G_{1}(x, t ; \lambda) v_{0}(t, \lambda) d t+f_{0}(x, \lambda), \quad(x, 0) \in I_{1}
$$

где

$$
\begin{gathered}
G_{1}(x, t ; \lambda)= \begin{cases}\frac{\operatorname{sh} \lambda x \operatorname{sh} \lambda(1-t)}{\lambda \operatorname{sh} \lambda}, & 0 \leq t \leq x, \\
\frac{\operatorname{sh} \lambda t \operatorname{sh} \lambda(1-x)}{\lambda \operatorname{sh} \lambda}, & x \leq t \leq 1,\end{cases} \\
f_{0}(x, \lambda)=\psi_{2}(0, \lambda)-x\left[\psi_{1}(1, \lambda)-\psi_{2}(0, \lambda)\right]+ \\
+\lambda^{2} \int_{0}^{1} G_{1}(x, t ; \lambda)\left\{\psi_{2}(0, \lambda)-t\left[\psi_{1}(1, \lambda)-\psi_{2}(0, \lambda)\right]\right\} d t .
\end{gathered}
$$

Используя решение задачи Коши для уравнения (7) в областях $D_{j}(j=\overline{1,3})$ с учетом (8), (9) получим функциональное соотношение между $\tau^{\prime}{ }_{j}(\omega, \lambda)$ и $v_{j}(\omega, \lambda)$, перенесенное из области $D_{j}$ на $I_{j}(j=\overline{1,3})$ :

$$
\begin{gathered}
\tau_{j}^{\prime}(\omega, \lambda)=P_{j}^{\prime}(\omega, \lambda)- \\
-(-1)^{j} \int_{\omega}^{\sigma_{j}} v_{j}(t, \lambda) \frac{\partial}{\partial \omega}\left\{I_{0}[\lambda(\omega-t)] d t\right\}+(-1)^{j} v_{j}(\omega, \lambda), \quad \omega \in I_{j},
\end{gathered}
$$

где $P_{j}(\omega, \lambda),(j=\overline{1,3})$ - заданные непрерывно-дифференцируемые функции, а $\omega=x, \sigma_{1}=1$ при $j=1, \omega=y, \quad \sigma_{2}=0$ при $j=2, \omega=y, \quad \sigma_{3}=1$ при $j=3$.

Так как $\tau_{0}(x, \lambda)=\tau_{1}(x, \lambda)$ то из соотношений $(27)$ и (30) исключив $\tau_{1}^{\prime}(x, \lambda)$, получим интегральное уравнение Фредгольма второго рода относительно $v_{1}(x, \lambda)$ :

$$
v_{1}(x, \lambda)+\int_{0}^{1} M_{1}(x, t ; \lambda) v_{1}(t, \lambda) d t=f_{1}(x, \lambda), \quad(x, 0) \in I_{1},
$$


где

$$
f_{1}(x, \lambda)=P^{\prime}{ }_{1}(x, \lambda)-f^{\prime}{ }_{0}(x, \lambda) .
$$

В силу свойств функций $G_{1}(x, t ; \lambda)$ и $I_{0}[\lambda(x-t)]$ заключаем, что $M_{1}(x, t ; \lambda)$ непрерывна в $\{(x, t): 0 \leq x<t, t<x \leq 1\}$, а функция $f_{1}(x, \lambda)$ принадлежать в классу $C^{2}(0,1)$. Отсюда следует, что уравнение (31) является интегральным уравнением Фредгольма второго рода.

Из теории интегральных уравнений Фредгольма второго рода и из единственности решения задачи $A_{1} T_{\lambda}$ следует однозначная разрешимость интегрального уравнения (31) в классе $v_{1}(x, \lambda) \in C^{2}\left(I_{1}\right)$, причем $v_{1}(x, \lambda)$ может иметь особенность порядка меньше единицы на концах интервала $I_{1}$.

Решая в области $D_{0}$ первую краевую задачу с условиями

$$
\begin{gathered}
u(x,+0, \lambda)=\tau_{1}(x, \lambda), \quad(x, 0) \in \overline{I_{1}} \quad u(+0, y, \lambda)=\tau_{2}(y, \lambda), \quad(0, y) \in \overline{I_{2}}, \\
u(+1, y, \lambda)=\tau_{3}(y, \lambda), \quad(0, y) \in \overline{I_{3}},
\end{gathered}
$$

для уравнения (7), убеждаемся, что решение $u(x, y, \lambda)$ можно представить в виде

$$
u(x, y, \lambda)=u_{0}(x, y, \lambda)+\int_{0}^{y} d \eta \int_{0}^{1} R_{1}(x, y ; \xi, \eta ; \lambda) u_{0}(\xi, \eta, \lambda) d \xi
$$

где $R_{1}(x, y ; \xi, \eta ; \lambda)$ резольвента ядра $-\lambda^{2} G_{1}(x, y ; \xi, \eta)$,

$$
\begin{gathered}
u_{0}(x, y, \lambda)=\int_{0}^{y} G_{1 \xi}(x, y ; 0, \eta) \tau_{2}(\eta, \lambda) d \eta+\int_{0}^{y} G_{1 \xi}(x, y ; 1, \eta) \tau_{3}(\eta, \lambda) d \eta+ \\
+\int_{0}^{1} G_{1}(x, y ; \xi, 0) \tau_{1}(\xi, \lambda) d \xi \\
G_{1}(x, y ; \xi, \eta)=\frac{1}{2 \sqrt{\pi(y-\eta)}}\left\{\exp \left\{-\frac{(x-\xi)^{2}}{4(y-\eta)}\right\}-\exp \left\{-\frac{(x+\xi)^{2}}{4(y-\eta)}\right\}\right\}+ \\
+\frac{1}{2 \sqrt{\pi(y-\eta)}} \sum_{\substack{n=-\infty \\
n \neq 0}}^{+\infty}\left\{\exp \left\{-\frac{(x-\xi+2 n)^{2}}{4(y-\eta)}\right\}-\exp \left\{-\frac{(x+\xi+2 n)^{2}}{4(y-\eta)}\right\}\right\},
\end{gathered}
$$

$G_{1}(x, y ; \xi, \eta)-$ функция Грина первой краевой задачи для уравнения $u_{x x}-u_{y}=0$.

Дифференцируя (33) по $x$, а затем, переходя к пределу при $x \rightarrow+0$ и $x \rightarrow 1+0, \mathrm{c}$ учетом (34), (30) получим

$$
\begin{gathered}
v_{2}(y, \lambda)+\int_{0}^{y} E_{1}(y, t) \nu_{2}(t, \lambda) d t+\int_{0}^{y} E_{2}(y, t) \nu_{3}(t, \lambda) d t=\Phi_{1}(y, \lambda), \\
v_{3}(y, \lambda)+\int_{0}^{y} E_{3}(y, t) \nu_{3}(t, \lambda) d t+\int_{0}^{y} E_{4}(y, t) \nu_{2}(t, \lambda) d t=\Phi_{2}(y, \lambda),
\end{gathered}
$$


где $E_{1}(y, t)\left[E_{3}(y, t)\right]$ функции непрерывные в $\{(y, t): 0 \leq t<y \leq 1\}$, а при $t \rightarrow y$ имеет слабую особенность[меньше единицы]. Функции $E_{2}(y, t)$ и $E_{4}(y, t)$ при $t \rightarrow y$ ограничены. Правая часть интегрального уравнения (35) и (36) принадлежит в классу $\Phi_{i}(y, \lambda) \in C[0,1] \cap C^{2}(0,1), \quad(i=1,2)$.

Отсюда следует, что интегральные уравнения (35) и (36) являются интегральными уравнениями типа Вольтерра относительно функций $v_{2}(y, \lambda)$ и $v_{3}(y, \lambda)$.

Согласно теории интегральных уравнений Вольтерра второго рода заключаем, что интегральные уравнения (35) и (36) однозначно разрешимы в классе $C[0,1] \cap C^{2}(0,1)$.

Таким образом, решение задачи $A_{1} T_{\lambda}$ можно восстановить в области $D_{0}$ как решение первой краевой задачи для уравнения (7) с условиями (32), а в областях $D_{j}(j=\overline{1,3})$ - как решение задачи Коши для уравнения (7) [10].

Следовательно, задача $A_{1} T_{\lambda}$ однозначно разрешима. Этим завершается исследование задачи $A_{1} T_{\lambda}$ для уравнения (7).

Теорема 4 доказана.

Теорема 5. Пусть решение $и(x, y, \lambda)$ плоской задачи $A_{1} T_{\lambda}$ для уравнения (7) существует и дается формулой

$$
u(x, y ; \lambda)=\frac{1}{\sqrt{2 \pi}} \int_{-\infty}^{+\infty} U(x, y, z) e^{i \lambda z} d z
$$

причем при больших значениях $|\lambda|$ допускает оценку (23). Тогда в области $\Omega$ решение задачи $A_{1} T$ для уравнения (1) существует и находится формулой (6) и наоборот.

Замечание 1. Оценка (23) обеспечивает существование интеграла (6), дающего решение задачи $A_{1} T$

Замечание 2. Используя свойства преобразования Фурье [14, С. 537], [15, С. 36], можно доказать справедливость оценок (4), т.е. $U(x, y, z), U_{x}(x, y, z), U_{y}(x, y, z), U_{z}(x, y, z)$ стремятся к нулю при $|z| \rightarrow \infty$ и существуют все соответствующие несобственные интегралы [4].

Замечание 3. Из теоремы 5 следует эквивалентность задач $A_{1} T$ и $A_{1} T_{\lambda}$. .

Конкурирующие интересы. Авторы заявляют, что конфликтов интересов в отношении авторства и публикации нет.

Авторский вклад и ответственность. Все авторы участвовали в написании статьи и полностью несут ответственность за предоставление окончательной версии статьи в печать. Окончательная версия рукописи была одобрена всеми авторами.

\section{Список литературы/References}

[1] Бицадзе А.В. Об одном трехмерном аналоге задачи Трикоми, Сибирский математический журнал, 3 (1962), 642-644.

[2] Нахушев А. М., “Об одном трехмерном аналоге задачи Геллерстедта”, Дифференциальнье уравнения, 4:1 (1968), 52-62.

[3] Салахитдинов М. С., Исламов Б., “О трехмерном аналоге задачи Трикоми для уравнения смешанного типа”, Доклады АН СССР, 311:4 (1990), 797-801.

[4] Пономарёв С. М., “К теории краевых задач для уравнений смешанного типа в трёхмерных областях", Докладь АН СССР, 246:6 (1979), 1303-1306. 
[5] Ежов А. М., Пулькин С. П., "Оценка решения задачи Трикоми для одного класса уравнений смешанного типа", Докладь АН СССР, 193:5 (1970), 978-980.

[6] Джураев Т. Д., Сопуев А., Мамажонов М., Краевые задачи для уравнений парабологиперболического типа, ФАН, Ташкент, 1986, 220 с.

[7] Исломов Б.И., Аликулов Е. К., "О трехмерном аналоге задачи Геллерстедта для нагруженного уравнения эллиптико-гиперболического типа”, Узбекский математический журнал, 2012, № 1., 61-73.

[8] Yuldashev T. K. , Islomov B. I. , Alikulov E. K., "Boundary-value problems or loaded thirdorder parabolic-hyperbolic equations in infinite three-dimensional domains", Lobachevskii Journal of mathematics, 41:5 (2020), 922-940.

[9] Islomov B. I., Umarova G. B., "Three-dimensional Problems for a Parabolic-Hyperbolic Equation with Two Planes of Change of Type", Lobachevski Journal of Mathematics, 41:9 (2020), 1811-1822.

[10] Умарова Г.Б., “Трехмерная задача Трикоми для параболо-гиперболического уравнения с двумя плоскостями изменения типа", Вестник Института математики, 2020, № 3, 153-166.

[11] Agmon S., Nirenberg L., Protter M.H., "A maximum principle for a class of hyperbolic equations and applications to equations of mixed elliptic-hyperbolic type", Communications on Pure and Appl. Math., 1953., 455-470.

[12] Ильин А. М., Калашников А. С., Олейник О. А., “Линейные уравнения второго порядка параболического типа”, ЖВМ и МФ, 4:6 (1965), 1006-1024.

[13] Салохитдинов М.С., “Об одной краевой задаче для уравнения третьего порядка смешанно-составного типа", Краевые задачи для неклассических уравнений математической физики, Фан, Ташкент, 1986, 3-16.

[14] Фихтингольц Г. М., Курс дифференциального и интегрального исчисления. Т. 3, Наука, М., 1970, 556 с.

[15] Самко С.Г., Кильбас А.А., Маричев О.И., Интеграль и производные дробного порядка и некоторые их приложения, Наука и Техника, Минск, 1987, 668 с. 
MSC 35M10, 35M12, 35L10, 35K10, 42B10

Research Article

\section{On a boundary value problem for a mixed equation with three planes of type change in an infinite prismatic domain}

\section{B. I. Islomov', G. B. Umarova ${ }^{2}$}

${ }^{1}$ National University of Uzbekistan named after Mirzo Ulugbek, 100174, University Street, 4. Tashkent, Uzbekistan

${ }^{2}$ Kokand State Pedagogical Institute named after Mukimi, 150700, Kokand, Uzbekistan, Fergana region, Kokand, st. Turon, 23, Republic of Uzbekistan.

E-mail: islomovbozor@yandex.com, guzalxon5111987@gmail.com

In this paper, in an infinite prismatic domain, one problem is formulated and studied for a parabolic-hyperbolic equation with three planes of type change. The main methods for studying the problem posed is the Fourier transform. The uniqueness and existence of a solution to the problem is proved.

Key words: equation with three planes of type change, Fourier transform, regular solution, extremum principle, solution estimation.

DOI: $10.26117 / 2079-6641-2021-34-1-19-28$

Original article submitted: 26.01.2021

Revision submitted: 06.03.2021

For citation. Islomov B. I., Umarova G. B. On a boundary value problem for a mixed equation with three planes of type change in an infinite prismatic domain. Vestnik KRAUNC. Fiz.-mat. nauki. 2021, 34: 1, 19-28. DOI: 10.26117/2079-6641-2021-34-1-19-28

Competing interests. The authors declare that there are no conflicts of interest regarding authorship and publication.

Contribution and Responsibility. All authors contributed to this article. Authors are solely responsible for providing the final version of the article in print. The final version of the manuscript was approved by all authors.

The content is published under the terms of the Creative Commons Attribution 4.0 International License (https://creativecommons.org/licenses/by/4.0/deed.ru)

(C) Islomov B. I., Umarova G. B., 2021

Funding. The study was carried out without financial support from foundations. 